\title{
REALIDAD DE LA PRÁCTICA PEDAGÓGICA EN TIEMPOS DE PANDEMIA
}

\author{
REALITY OF PEDAGOGICAL PRACTICE IN TIMES OF PANDEMIC \\ (iD) Carelis B Sánchez Bozo ${ }^{*}$, (D) Elodou Isea ${ }^{1}$, (D) Elizabeth Vera ${ }^{1}$ \\ carelisanchezb@gmail.com; elodou_24@hotmail.com; viveca_vera@hotmail.com \\ 1Universidad Nacional Experimental Rafael María Baralt, Venezuela
}

*Correspondencia: Carelis B Sánchez Bozo. Email: carelisanchezb@gmail.com

Recibido: 21.04.21 | Aprobado: 01.07.21

\section{RESUMEN}

El objetivo fue analizar la realidad de la práctica pedagógica y conocer cuál ha sido el giro que la pandemia ha ocasionado en las instituciones de educación media; para lograrlo se utilizó la metodología cuantitativa con la aplicación de estadística descriptiva. Evidenciando que el 66,67 \% no acceden a integrar en su pedagogía las estrategias metodológicas propias de la pedagogía tecnológica mientras el 77,78\% aseguran no asumir los nuevos métodos pedagógicos dentro de la dinámica formativa. Se concluyó que la pedagogía desarrollada está apegada al paradigma presencial solo con el cambio de los recursos y medios. La complejidad de la situación se relaciona más con la disposición e interés de las instituciones que deben internalizar el logro de la calidad educativa mediante la innovación.

Palabras clave: pedagogía, tecnológica, educación, pandemia.

\begin{abstract}
The objective was to analyze the reality of the pedagogical practice and to know what has been the turn that the pandemic has caused in the institutions of secondary education; To achieve this, the quantitative methodology was used with the application of descriptive statistics. Evidence that $66.67 \%$ do not agree to integrate the methodological strategies of technological pedagogy into their pedagogy while $77.78 \%$ claim not to assume the new pedagogical methods within the formative dynamics. It was concluded that the pedagogy developed is attached to the face-to-face paradigm only with the change in resources and means. The complexity of the situation is more related to the willingness and interest of the institutions that must internalize the achievement of educational quality through innovation.
\end{abstract}

Keywords: pedagogy, technology, education, pandemic. 


\section{INTRODUCCIÓN}

Etimológicamente la Pedagogía tiene su origen la palabra griega paidagōgeō; en la cual paidos significa niño, y ágō significa guía, se trata en todo caso de dirigir al niño. Apoyado en esta concepción primigenia, el docente, ha de enfocarse en un ejercicio, un análisis crítico y reflexivo de la educación a través de fundamentos teóricos, sustentado en metodologías específicas y técnicas especiales. Por lo que su labor no se circunscribe solamente a la docencia, sino que involucra la planeación y gestión de planes educativos particulares, creación de materiales didácticos, asistencia del educando con problemáticas de aprendizaje y se dedica al diseño de programas que impulsen el desarrollo educacional de las entidades para fines sociales.

Sin embargo, como toda gestión, para perdurar en el tiempo, el docente debe ser un constante estudioso de su realidad para lograr la adaptación a las situaciones, por complejas que estas puedan resultar, por ello siempre ha de prepararse atendiendo al contexto y siendo visionario en la búsqueda de una calidad educativa. Aun así, históricamente, nunca hubo un hecho para considerar un cambio tan radical de los procesos, debido a que la humanidad, no había confrontado una complejidad como la desatada en el año 2020 con la diseminación de la pandemia, lo que provocó una ruptura del trayecto educativo como proceso integrado.

Tal como lo plantea el Informe Covid-19, la pandemia de enfermedad por coronavirus (COVID-19) ha provocado una crisis sin precedentes en todos los ámbitos. En la esfera de la educación, esta situación de global afectación dio lugar al cierre masivo de las actividades presenciales de instituciones educativas en más de 190 países, todo ello para evitar la propagación del virus y mitigar su impacto. Según datos de la Organización de las Naciones Unidas para la Educación, la Ciencia y la Cultura (UNESCO), a mediados de mayo de 2020 más de 1.200 millones de estudiantes de todos los niveles de enseñanza, en todo el mundo, habían dejado de tener clases presenciales en la escuela. De estos, más de 160 millones eran estudiantes de América Latina y el Caribe. (Cepal Unesco 2020),

Como respuesta inmediata, todos los sistemas educativos estudiaron alternativas y emprendieron múltiples esfuerzos para enfrentar lo acontecido, siendo el común denominador de estas acciones, el fortalecer las interacciones entre docentes, alumnos, padres, representantes y demás integrantes de la escuela y comunidad; con miras a enriquecer la labor docente en la formación y capacitación en el proceso enseñanza aprendizaje. Es importante resaltar, que los cambios iniciales en este proceso fue el de actitud en los educadores para enfrentar situaciones de aprendizaje de manera óptima y efectiva, aun cuando percibía que la nueva operación no mostraba facilidades claras.

Sobre este particular, para el caso específico del docente, la complejidad, reveló en mayor grado algunas debilidades en su desempeño, que posiblemente no eran percibidas debido a que en la modalidad presencial, no eran tan evidentes, pero en el escenario totalmente virtualizado, su escasa pericia lo limita para gestionar el proceso de enseñanza, desde lo más elemental aspecto pedagógico. Afianzando lo anterior, se tiene que los docentes en su mayoría, no contaba con entrenamiento previo relacionado con la utilización de recursos digitales, para impartir lecciones totalmente de forma sincrónica y/o asincrónica; en consecuencia, esto generó en una serie de espacios de aprendizaje, 
donde era necesario comprender cómo la mediación pedagógica, podía instrumentarse fuera del contexto educativo más común, el aula. (Carranza-Marchena 2020).

Resulta evidente, que el docente quedó inmerso dentro de un cambio radical, con un espacio de trabajo transformado incluyendo sus recursos, su medio de soporte y en general un cambio contextual, similar situación vivencian los estudiantes. Como fenómeno, este hecho ha sido estudiado por diversos investigadores, encontrando que la experiencia virtual define la realidad espacial de los nuevos entornos educativos, y por ende todos los elementos que la constituyen deben adaptarse. (Espegel-Alonso; FelizRicoy; Buedo García 2020).

En dirección a enmarcar, toda interacción dentro de una serie de medidas de bioseguridad, destinadas a preservar la salud y evitar los contagios en las instituciones educativas, se impacta todo lo que representa valor en la educación, como es el caso de la pedagogía, acción destinada al planteo, estudio y solución del problema educativo, de una forma más directa es un conjunto de normas, leyes o principios que se encargan de regular el proceso educativo. (Michelle 2006)

Es por ello, que la responsabilidad del docente ante la acción pedagógica, en su modo presencial dentro del aula, le permitía identificar la fluidez del proceso formativo, conocer la manera en la cual cada estudiante reacciona ante la facilitación de las ideas y la asignación de las actividades, pero dentro de este escenario virtual e inafectivo por naturaleza, es más complicado y existe preocupación al pensar, que el trabajo pedagógico interfiera esa atención, al interponer medios que hacen muy rígida la interacción.

En este contexto, la educación comienza a verse cuestionada de múltiples maneras, hoy en día los docentes deben hacer un alto y replantear sus actuales caminos, pensar en aquellos no acostumbrados y así poder integrarse a la acción pedagógica transformada permitiendo planificar estrategias innovadoras significativas, así como relevantes para el desarrollo de los contenidos curriculares implementados a través de un modelo mediado por la tecnología en sus diversas formas.

En este sentido, experiencias como las planteadas en la investigación titulada COVID-19: Frente al desafío pedagógico de lo presencial a lo virtual (2020), refirió como objetivo el analizar el desafío pedagógico que enfrenta la educación en relación a la pandemia del COVID-19, logrando iniciar con una premisa donde, la nueva modalidad educativa, que inserta la virtualidad llevó a los estudiantes, docentes y directivos a realizar serios cuestionamientos en el que se aquejan entre ellos mismos, pudiéndose concebir una resistencia al cambio.

Señala el estudio en cuestión, que muchos actores, no utilizan la tecnología de manera adecuada, se habla de una actividad que debe hacerse a través de una computadora o aparatos móviles, pero siempre existe una excusa para no integrarse a la experiencia educativa virtual, mostrando desinterés; no todos en la misma proporción, pero al final incidente. Esta fue una condición que experimentó en el momento del trabajo presencial, pero, aun así, marca una brecha digital existente desde hace mucho tiempo.

En todo caso esto determina que estos docentes pueden estar usando la tecnología, pero en verdad no la están aprovechando por lo cual se subutiliza y se aprovecha en ese 
mismo sentido. En base a esto, los actores educativos, necesitan cambiar su actitud y disposición, para afrontar este nuevo desafío, se debe estar convencida de la calidad, la efectividad, modalidades mediatizadas por la virtualidad que se realiza.

Concluye este trabajo, que es necesario que el sector de la educación, plante recomendaciones para mitigar el impacto que ocasiona la epidemia que hoy afecta al mundo, y sensibilizar los riesgos de la enfermedad, por ello, están llamados a reinventarse pedagógicamente, haciendo lo que mejor saben hacer dentro de su ámbito educativo, pero de forma diferente, a través de estrategias, las cuales involucren el uso de las tecnologías y actividades virtuales, impulsando de esta manera la práctica del conocimiento, el trabajo autónomo y asistido. (Bracho y Bracho 2020).

De igual manera en el trabajo titulado Fortalezas y debilidades de la pedagogía en el ciberespacio en época de cuarentena COVID-19 (2020), logró plantearse como objetivo, el analizar los pros y los contras, que han surgido con el uso de la pedagogía en el ciberespacio, la misma que se ha tenido que implementar inesperadamente, por la crisis sanitaria que se vive, dando a conocer las fortalezas y debilidades que este enfoque está causando en la comunidad educativa.

Enfatiza que, la pedagogía en el ciberespacio es nueva, y destaca que existen docentes que realizan su mejor esfuerzo con el fin de adaptarse a esta nueva realidad, consultando nuevas estrategias tecnológicas y mostrándose dispuestos, a adaptarse a estos cambios, aun cuando esta misma realidad, ha impactado de manera contundente las posibilidades del docente, al no tener la pericia y experiencia suficiente como para integrarse a estas acciones innovadoras, y por ello, se expone a situaciones estresantes y complejas, que también afectan la posibilidad de una educación de calidad. (Prieto, F., Rodríguez, L., \& Verdugo, R. 2020).

De igual manera, otra importante consideración en este ámbito, plantea que en el trabajo pedagógico, los cambios de paradigmas representan una fuerte conversión en sus concepciones, diseños educativos, didáctica, práctica e incorporación a los ambientes virtuales y a la creación de entornos personalizados de aprendizaje, que se debe asumir tanto por docentes como estudiantes, los cuales deben "aprender, desaprender y reaprender", con el gran reto que implica en los ambientes de aprendizaje la inclusión de nuevos recursos de las TIC, que pueden estar mediados, preferiblemente, por dispositivos móviles. (Vidal Ledo M, Gavilondo Mariño X. 2018)

En dirección a ello, es necesario ver la virtualización como una oportunidad que acorta las distancias, enriquece el proceso de enseñanza aprendizaje, permite el desarrollo de los programas de estudio normalmente, sin necesidad de suspender clases, porque los materiales y las actividades permanecen accesibles todo el tiempo y revelan la actitud de los estudiantes ante la gestión de su conocimiento, fortalecen las relaciones e inculcan la colaboración entre todos los actores.

Sin embargo, el campo de la pedagogía, al igual que el resto de los componentes, debe generar una migración inteligente, que permita asumir el nuevo espacio, las herramientas, las estrategias, de manera que, se logre dar una conducción propia, 
procurando mantener los elementos fundamentales del proceso de formación de los estudiantes y mantener la calidad de trabajo del docente, como gestor de la enseñanza.

Por ello, el propósito de este artículo está centrado en analizar la realidad de la práctica pedagógica y conocer cuál ha sido el giro que la pandemia ha ocasionado, de manera que sea posible, encontrar las explicaciones de lo acontecido, permitiendo a su vez observar los ejercicios que resulten necesarios, para llevar a la pedagogía hacia una estrategia coherente con los tiempos y las demandas. Dentro de los aspectos más notables encontrados en la misma se tiene que el rol docente para este ejercicio pedagógico es el de ser un mediador entre el conocimiento y el estudiante, creando material que haga dinámico este proceso.

\section{MATERIALES Y MÉTODOS}

El ámbito de estudio se ubica en Instituciones de Educación Media ubicadas en la ciudad de Maracaibo, Venezuela, las cuales disponen del uso de plataformas tecnológicas como Classroom; la población estuvo conformada por docentes en diferentes áreas de conocimiento. De igual manera se trató de un estudio no experimental descriptivo, en específico se logró contar con un total de 80 docentes, seleccionándose una muestra de 42 a partir de una participación voluntaria por lo cual adquiere la condición de muestra intencional. (Hernández, Fernández y Baptista 2014).

Para el trabajo de la recolección de la información se utilizó un instrumento desarrollado por los investigadores, el mismo fue debidamente validado por dos expertos de la UNERMB en el ámbito metodológico y de contenido, ambos docentes de planta, con nivel doctoral. Se trató de una validación de contenido, por lo que se realizaron ajustes a las redacciones de algunos ítems. En cuanto a la confiabilidad se practicó una prueba piloto con 10 docentes que no formaron parte del estudio, de manera que los resultados permitieron calcular el coeficiente de Cronbach logrando un valor $\mathrm{rtt}=0,92$, lo que expresa su alta confiabilidad. Dicho instrumento, permitió conocer el comportamiento de la pedagogía que en la actualidad el docente ejecuta mediante el uso de las tecnologías a raíz de la circunstancia de pandemia, una vez obtenidos los datos, se procesó con estadística descriptiva.

\section{RESULTADOS}

En la obtención de los resultados se procedió a la aplicación del instrumento desarrollado para esta investigación, el cual estuvo dirigido a los docentes de instituciones de educación media, específicamente en aquellas donde la utilización de plataformas como Classroom permiten la interacción con los estudiantes, de manera que los aspectos relacionados con la pedagogía pudieran ser identificados dentro del ámbito de la tecnología. Estos resultados se presentan de manera resumida con su respectiva explicación en base al comportamiento de este en la realidad consultada para el ejercicio pedagógico del docente.

\section{Dimensión Estrategias Metodológicas}

\section{Figura 1.}

\section{Estrategias Metodológicas}




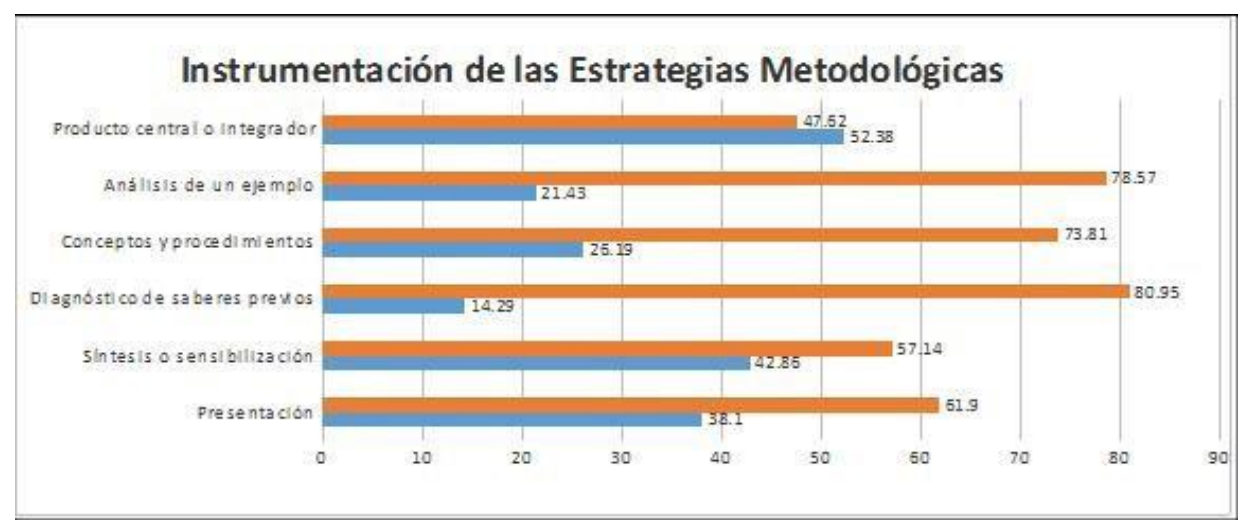

Fuente: Elaboración propia (2021)

En este particular al precisar las opiniones de los consultados, se abordó la dimensión Estrategia Metodológica en la Figura 1, donde se evidenció que para el 38,10\% (16 docentes) se está acudiendo al uso de la Presentación dentro del ejercicio pedagógico, por lo que algunos docentes en las interacciones formativas con los estudiantes, pueden generar interrogantes para ilustrar la necesidad de estudio sobre el tema, a su vez, para una menor cantidad de docentes, es posible conducir una socialización contextualizada con la realidad del momento, para lograr un aprendizaje significativo en los estudiantes, finalmente, en su mayoría, estos no disponen de recursos como el video, para exponer este tipo de estrategia a los estudiantes.

Con relación al indicador Síntesis o sensibilización, se evidencia que 42,86\% de los docentes, tienen la posibilidad de asumir esta estrategia metodológica dentro de su desempeño pedagógico, el 57,14\% no logra tal posibilidad, pero esto se identifica cuando los mismos aseguran, que tienen limitaciones para conocer los elementos que motivan a los estudiantes en el logro de sus actividades, posiblemente se debe, a la baja interacción síncrona que se presenta, de igual manera, en su mayoría consideran que, pocas veces pueden incorporar expresiones motivadoras, en el mensaje que exponen a sus estudiantes para contrarrestar la complejidad de la pandemia, a la par, que no tienen manera de realizar videos, para llevar este tipo de mensaje en sus interacciones con los estudiantes.

En base a los resultados del indicador Diagnóstico de saberes previos, se observa cómo el 14,29\% de los docentes, logran incorporar esta estrategia en su gestión pedagógica, mientras que el $80,95 \%$ no está en esa oportunidad. Al respecto, se tiene que, en algunas oportunidades, los docentes pueden facilitar a sus estudiantes casos o ejemplos para que ellos evidencien, el tema en el cual se incursiona, sin embargo, son pocos los que pueden realizar una socialización, la cual les permita formular preguntas, para así promover una retroalimentación. En esta misma línea de ideas, aseguran que, no utilizan recursos como cuestionarios con sus estudiantes, para obtener este tipo de información útil para retroalimentarse.

Dentro de los hallazgos, se tiene para el caso del indicador Conceptos y procedimientos, que para el 26,19\% de los docentes, es posible asumir este tipo de estrategia metódica dentro de su accionar pedagógico, y el 73,81\% considera que les resulta complejo incorporarla dentro de su trabajo pedagógico. Al respecto, para los docentes consultados, ellos por lo general no logran presentar a los estudiantes los 
aspectos clave que han de integrar para el aprendizaje de los contenidos manejados, además, son pocos los que pueden brindarles respuestas a las diversas preguntas, que se formulan los estudiantes de una manera asíncrona. Sumado a esto, no tienen manera todos para generar videos cortos para cumplir con los propósitos descritos.

En el caso del indicador Análisis de un ejemplo, 21,43\% de los docentes, consideran que pueden asumir este tipo de estrategia en su gestión o desempeño pedagógico, pero contrario a ello, 78,57\% de estos, niegan tal oportunidad. De ahí que, en lo acontecido dentro de estas instituciones de educación media que hacen uso de recursos como el Classroom, son pocos los docentes, que pueden facilitar ejemplos a los estudiantes a manera de guía, en la elaboración de las actividades, además, no todos, pueden brindar explicaciones a los estudiantes, para el desarrollo de las asignaciones en su área de conocimiento, esto debido a la carencia que la mayoría tiene para utilizar sistemas de video conferencia para afianzar esta labor.

Con relación al indicador Producto central o integrador, los resultados expresan que 52,38\% de los docentes incorporan esta estrategia dentro del aspecto pedagógico que manejan en las instituciones ante la situación de pandemia, solo el 47,62\% no están en esa oportunidad. Sobre este particular, la mayoría de estos docentes, plantean a sus estudiantes la utilización de normas de trabajo o instrucciones para la elaboración de las asignaciones, sin embargo, pocos ejecutan el proceso de evaluación mediante el uso de una rúbrica socializada, además, algunos disponen de notas de voz, para brindar las orientaciones necesarias.

Al totalizar el comportamiento de la dimensión Estrategia Metodológica, se hace evidente que el 32,54\% de los docentes, acceden a incorporar las mismas dentro de su haber pedagógico en el trabajo que se desarrolla mediante la virtualización de las actividades de enseñanza y aprendizaje de los estudiantes en las instituciones de educación media que cuentan con apoyo tecnológico de plataformas, mientras que el 66,67\% de los docentes, descartan que las mismas sean parte de su accionar pedagógico.

\section{Dimensión Nuevos métodos pedagógicos}

\section{Figura 2:}

Nuevos métodos pedagógicos

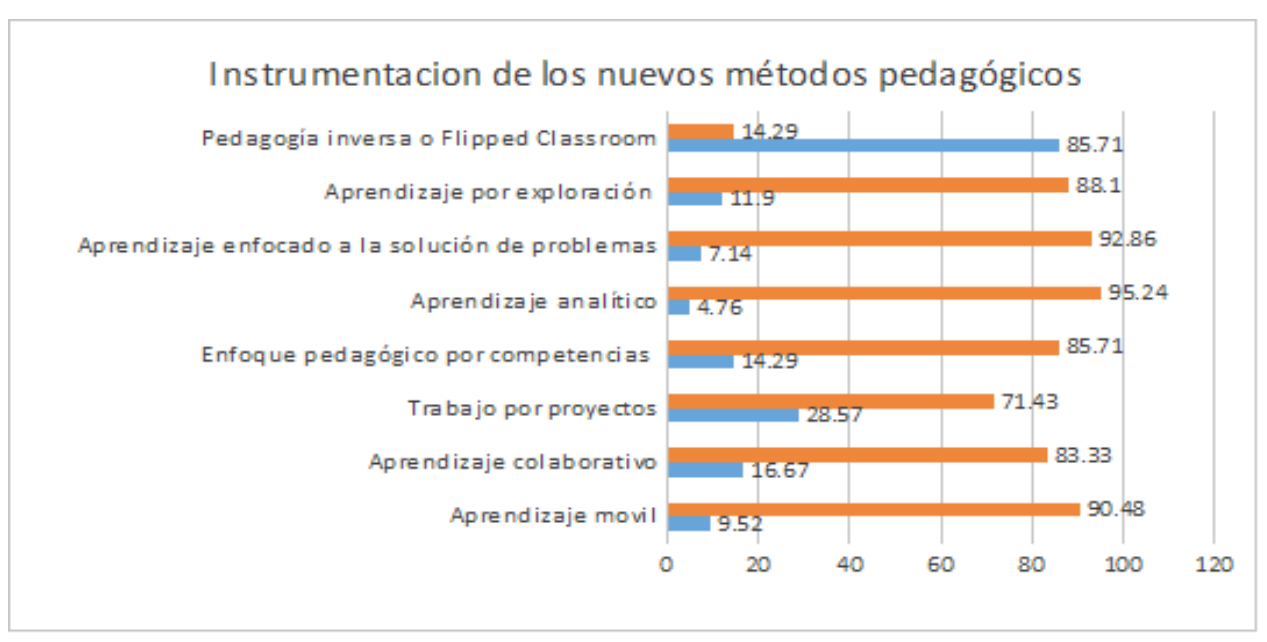


Fuente: Elaboración propia (2021)

Para el caso de la dimensión Nuevos métodos pedagógicos, mostrados en la Figura 2, la consulta también permitió conocer a través de la opinión de los docentes, como se presenta la realidad de cada indicador, por ello, en el caso del indicador Aprendizaje móvil, solo el 9,52\% del personal consultado considera asume este método pedagógico, aun cuando el 90,48\% de estos lo descarta, en base a esta consideración, señalan en su mayoría que no pueden crear oportunidades de aprendizaje en cualquier contexto, mediante el uso de las TIC, por lo que tampoco, están posibilitados de romper las limitaciones espaciotemporal, para lograr el proceso de enseñanza y aprendizaje, es decir, lograr que aprendan aun fuera de la posible interacción.

De los resultados alcanzados para el indicador Aprendizaje colaborativo, se establece para el 16,67\% de los docentes, este método pedagógico, forma parte de su trabajo dentro del proceso de enseñanza que activa mediante el uso de las tecnologías, sin embargo, el 83,33\% de estos, descarta se esté alcanzando esta oportunidad. Aseguran, que, aun disponiendo de servicios digitales en la plataforma, no les es posible, activar procesos de enseñanza aprendizaje, de manera conjunta con sus estudiantes, debido a la carencia de momentos síncronos con estos, o de generación de acuerdos, por lo tanto, la mayoría de ellos, aseguran que su pedagogía considera y logra compartir recursos digitales en la gestión educativa.

Con relación al indicador Trabajo por proyectos, en la realidad descrita por los docentes, para el 28,57\% es posible incorporar ese método pedagógico, aun cuando para el $71,43 \%$ sea contraria la alternativa de trabajarlo, como parte de la pedagogía que caracteriza el proceso en momentos de pandemia. Es por ello, que para algunos docentes se hace posible organizar procesos de aprendizaje, en torno a la consecución de unos objetivos en las asignaciones establecidas, pero contrario a esto, la mayoría señala que no puede asegurar que su práctica una pedagogía, está basada en la cooperación, mediante el uso de las TIC, pues solo, se están operando de manera instrumental.

$\mathrm{Al}$ conocer los resultados del indicador Enfoque pedagógico por competencias, la realidad descrita por los docentes señala que el 14,29\% consideran que pueden integrar este método pedagógico a la realidad del trabajo formativo en época de pandemia, aun cuando el 85,71\% niega tal integración, por considerar que no se encuentra esa oportunidad dentro de la condición que ofrece la institución. En este sentido, la mayoría de los consultados considera, que su accionar pedagógico, no está orientado a aumentar las capacidades de los estudiantes, a la par de descartar, que pueda definir estrategias de organización de las competencias según el nivel requerido por el nivel formativo.

Se destaca en el caso del indicador Aprendizaje analítico, que 4,76\% de los docentes consultados, señalan que pueden integrar este método pedagógico en su gestión educativa durante el tiempo de pandemia con la asistencia de las TIC, aun cuando el 95,24\% de estos descarta que eso sea posible. Precisan los docentes, que no tienen oportunidad para conformar materiales digitales, para que el estudiante acceda a estos en la evaluación de su rendimiento, mientras que, se descarta casi por completo que utilicen programas que evalúen al estudiante, favoreciendo la mejora constante de las estrategias de instrucción virtuales. 
De acuerdo a los resultados del indicador Aprendizaje enfocado a la solución de problemas, donde $7,14 \%$ de los docentes asegura que asumen las pautas de este método, pero contrario a ello, 92,86\% refiere que no les es posible tal oportunidad, por ello exponen que no tiene posibilidades, de organizar actividades destinadas a solventar un problema, que se presenta para la consecución de unos objetivos previos, de igual manera, la mayoría descarta que estén desarrollando una pedagogía como un sistema de análisis donde la clave es el pensamiento activo del estudiante.

En función a los resultados del indicador Aprendizaje por exploración, donde $11,9 \%$ de los docentes consultados, consideran que asumen el método descrito, aun así, $88,10 \%$ descartan que esto sea posible, en base a esto, aseguran que no propician la curiosidad innata del estudiante, creando condiciones adecuadas para explorar la realidad, además aseguran, no poder aprovechar las TIC para ampliar el campo de exploración en el proceso educativo.

Destacan en los resultados del indicador Pedagogía inversa o Flipped Classroom, que para el $85,71 \%$ de los docentes, refieren que se integran en esta metodología gracias a la infraestructura que tecnológicamente posee la institución, 14,29\% refiere no estar dentro de esa tendencia, debido a sus conocimientos básicos sobre el sistema, además, no puede aprovechar el potencial de esta. Es por ello por lo que, le es posible registrar las lecciones magistrales en la plataforma, en la cual se distribuyen a los estudiantes, sin embargo, descartan en su mayoría, que propicien actividades de reflexión de los conocimientos adquiridos en los procesos de formación.

\section{DISCUSIÓN}

El propósito del articulo está centrado en analizar la realidad de la práctica pedagógica en tiempos de pandemia, tomando en consideración que luego de transitado un año, desde el cual las actividades formativas, cambiaron de manera significativa su formato de trabajo, el docente, por ser uno de los actores esenciales en el proceso formativo, ha tenido que pasar a través de una serie de situaciones, en las cuales ha evolucionado y adaptado su gestión, y resulta de interés para la conducción del proceso educativo, conocer cómo se encuentra el aspecto de la pedagogía.

Desde una visión amplia, la realidad percibida resulta consistente con planteamientos que desde un análisis crítico señalan que la pandemia colocó en evidencia una serie de situaciones, para las cuales el sistema no estaba preparado, es decir, mostró la falta de preparación y oportunidad para el verdadero aprovechamiento de las tecnologías, las cuales fueron utilizadas en la mayoría de los casos en una forma instrumental.

Sin embargo, es de reconocer que los docentes, acostumbrados en su mayoría a las clases tradicionales, han tenido que reinventarse, para poder acercarse a los estudiantes, algunos con elementos básicos, como un teléfono móvil, con conexiones precarias de Internet, y otros con estudiantes que tenían todos los recursos tecnológicos para poder acceder a una clase en línea. (Peñafiel 2020).

Abordando la realidad estudiada, mediante la experiencia investigativa, en función a la dimensión Estrategia Metodológica, se encontró que, los docentes en su mayoría no 
asumen que el proceso educativo que ellos emprenden dentro de las instituciones, se ajuste a este componente esencial, de una pedagogía caracterizada por el uso de las tecnologías, por lo que, es claro que no se logra una alineación con la necesidad de cambios de paradigmas en sus concepciones, diseños educativos, didáctica, práctica e incorporación a los ambientes virtuales y a la creación de entornos personalizados de aprendizaje (Vidal Ledo M, Gavilondo Mariño X. 2018).

Habiendo estudiado lo relacionado al indicador Presentación, dicha estrategia determina, la necesidad de presentar a los estudiantes, mediante el uso de las Tic, una serie de planteamientos por parte del docente, donde se les formule interrogantes que han de servirle a este para definir una visión del conjunto, con el cual interactúa y además sopesar en qué condiciones se encuentra el dominio de sus conocimientos, de la misma, forma ha de facilitar la socialización de las realidades del proceso educativo, de manera que los estudiantes, puedan ser diligentes para confrontar la nueva manera de conducirse en el proceso. De acuerdo con los planteamientos de los docentes, esto no ha sido realizado con la regularidad que exige esta nueva pedagogía tecnológica, solo algunos docentes aseguran que, por motivación propia o por solicitud de la institución, promovieron algunas acciones.

Por ello, es importante reconocer que esta manera, en la que se cumple con la referida estrategia metodológica, como es la presentación, no está plenamente direccionada a la socialización del reto propuesto, por medio de una pregunta contextualizada, que evidencie la necesidad o problema, y en lo descrito por los docentes, se hace más evidente que no disponen de medios como el vídeo o infografías, que podrían ser portadores de la idea, razón que determina una diferencia con el criterio de Manrique (2020).

En el caso de la estrategia de Síntesis o sensibilización, los resultados exponen que para los docentes consultados, la posibilidad de conocer los elementos que motivan a los estudiantes, en el logro de sus actividades, no se encuentra tan a la mano, y aunque algunos aseguran que pueden estimular este componente, la realidad es que no se ajusta a la realidad imperante, por lo que la acción, no supera la cotidiana intención del docente, para impulsar el logro de sus estudiantes, pero ahora es más complejo, pues la interacción se logra escasamente. Esto determina una diferencia con lo señalado en su concepción teórica hace énfasis en promover la motivación frente al trabajo a realizar. (Manrique 2020).

Esto también, puede relacionarse con el hecho que, la nueva realidad obliga al sujeto a cambiar la interacción social física a una virtual, y el ambiente educativo por el del hogar, aislándolos del clima educativo, manejado durante todo este tiempo de manera presencial, donde el docente y compañeros de clases, servían guías y apoyo en las labores académicas, por lo que el estudiante ha experimentado sentirse extraviado, con la necesidad de buscar compañeros o docentes que lo guíen en algunas tareas, lo que aumenta la dificultad de desarrollarla y el factor de riesgo para la conducta desertora. (Galindo Cuervo, N. y Vela Palacios, J. 2020).

La realidad del indicador Diagnóstico de saberes previos establece una diferencia de lo acontecido por los docentes y el criterio que expone, que es necesario que el 
estudiante obtenga del docente casos que evidencien el tema, a partir de una socialización, que puede darse mediante la formulación de preguntas y retroalimentación, pero lo señalado también dista, en el uso de medios como cuestionarios, test, foros entre otros. (Manrique 2020). En la realidad de estas instituciones, no se tiene la oportunidad para que el docente pueda instrumentar esta acción como parte de esta nueva manera de llevar la pedagogía tecnológica, y por lo tanto determina, que se está procediendo de manera deficiente para el logro educativo.

Dentro de los aspectos que caracterizan la situación del docente en su ejercicio pedagógico, el indicador Conceptos y procedimientos, mostró que no se cumple el principio donde el docente, expone e insiste al estudiante lo mínimo aprender, basando esa Interacción a través de preguntas además de utilizar vídeo para llevar esa información. En la realidad de estos profesionales, el acceso a la generación de vídeos es baja, muchos no disponen de los medios como generarlos y las instituciones por su parte se muestran muy exigentes para la elaboración de este. Por ello no se logra una alineación a los principios teóricos que lo orientan para la pedagogía tecnológica. (Manrique 2020).

En el caso del indicador Análisis de un ejemplo, las experiencias de los docentes señalaron que lo consolidado de los materiales y las actividades, les limita para facilitar a los estudiantes un ejemplo, que sirva de guía en la elaboración de las asignaciones y además muestre la aplicación del tema que se estudia. Esto es determinante, debido a que estos profesionales por lo general conforman materiales digitalizados donde la oportunidad de acceder a la video conferencia es mínima, no permitiendo que esta alternativa se consolide dentro del proceso actual.

Finalmente, para el indicador Producto central o integrador, lo establecido en la realidad de los docentes, expone que ellos logran incorporar el uso de normas para la realización de las actividades, por lo que es uno de los pocos puntos en los cuales se incorpora la estrategia. Todas estas situaciones determinan un panorama que posiciona al docente dentro de una pedagogía tradicional, que solo utiliza a las tecnologías como un vehículo, pero es menester comprender que toda acción pedagógica, debe ser no solo planificada por la institución y sus docentes, sino que, además, para integrarse en el medio tecnológico, es más comprometedor este vínculo para hacerlo tangible y en este caso no se está cumpliendo.

Para el caso de la dimensión Nuevos métodos pedagógicos, las realidades en las que se sitúan los docentes, determinan que los mismos tienen una mínima presencia en la realidad pedagógica, por lo que no son parte de un esquema centrado en el aprovechamiento de las tecnologías. Por ello, esta manera en la cual se ha presentado la realidad del ejercicio pedagógico, no se inserta en la consideración que la práctica en las aulas virtuales requiere como el influjo de dos grandes vectores de cambio: a) los cambios instrumentales y tecnológicos, es decir, la incorporación de nuevos útiles didácticos; y, b) la evolución de las teorías pedagógicas, los cuales son momentos cruciales y se retroalimentan en algunos efectos. Muchas de estas corrientes metodológicas, provienen de teorías y sistemas anteriores a la aparición de Internet y renovada actualidad (Gabinete de Comunicación y Educación de la Universidad Autónoma de Barcelona 2014). 
En cuanto a lo expuesto por los docentes, estos argumentan para el indicador Aprendizaje móvil, descartan que puedan utilizar todas las posibilidades ofrecidas por las TIC para crear oportunidades de aprendizaje en cualquier contexto, con independencia del momento y del lugar. (Gabinete de Comunicación y Educación de la Universidad Autónoma de Barcelona 2014). Esto no se encuentra favorecido, debido a que el docente aun con la oportunidad que tiene para trabajar desde su hogar, no posee conocimientos sólidos, ni las herramientas necesarias para con este compromiso, por ello, no accede en firme desde este aspecto ocasionando una brecha real para con la pedagogía tecnológica.

Basados en los resultados alcanzados en el indicador Aprendizaje colaborativo, se tiene que los docentes no logran acciones constantes, para emprender procesos de enseñanza y aprendizaje basados en un trabajo conjunto, entre quienes conforman el entramado educativo, apoyados por los distintos servicios digitales a los cuales solo tienen acceso efectivo dentro de las instituciones, por la carencia de acceso a Internet que pueden tener desde sus hogares, así como por el costo que esto representa, por tratarse de servicios poco accesibles, al igual que el servicio de datos, que pueden obtener mediante el uso de teléfonos celulares.

Los hechos descritos dentro de la gestión de la pedagogía del docente demuestran que la misma no asume las definiciones planteadas para este indicador en su concepción teórica. (Gabinete de Comunicación y Educación de la Universidad Autónoma de Barcelona 2014). De igual manera, al estar dentro de esta realidad, la gestión pedagógica del docente no aprovecha del aprendizaje colaborativo aspectos como; la comunicación, captar la atención, desarrollar competencias transversales que propician la generación de habilidades sociales, la resolución de problemas, la autonomía, asumir responsabilidades; desarrollar la capacidad para reflexionar y tomar la iniciativa en distintas situaciones (Carrió, 2007; Plomp y Voogt, 2009; García-Valcárcel y Tejedor, 2010; Lee y Tsai, 2013)

Dentro de los hechos descritos por los docentes que caracterizan su desempeño pedagógico, se tiene que en el indicador Trabajo por proyectos, mostró una escasa posibilidad de estar siendo incorporado dentro de las actividades de formación, apoyadas en el uso de la tecnología, debido a las limitaciones que posee en el manejo de estas, además de la complejidad que confronta, al tener que generar actividades esencialmente individuales para los estudiantes.

Esto deja una clara diferencia con lo postulado para el indicador, del cual se tiene que se trata de una pedagogía que requiere el trabajo cooperativo, donde se organizan los procesos de aprendizaje, en torno a la consecución de unos objetivos propios de la realización de un proyecto, organizado en tareas u operaciones de índole pragmática e intelectual. Gabinete de Comunicación y Educación de la Universidad Autónoma de Barcelona 2014).

Basado en los resultados logrados en el indicador Enfoque pedagógico por competencias, la realidad expone que, la acción pedagógica del docente la cual se orienta por las consideraciones expresas en los contenidos programáticos y en la planificación que debe generar en la circunstancia de pandemia, dista de una posibilidad real para una transformación de las habilidades del estudiante, la escasa interacción limita brindar el 
respaldo necesario ante inquietudes o dudas de los jóvenes, por lo que no existe un verdadero direccionamiento hacia el impulso de las competencias señaladas.

Estos hechos, relacionados con el indicador antes descrito, no se encuentran en consonancia con su definición, en la cual el enfoque dentro de la perspectiva de los sistemas educativos ha de estar orientado, a aumentar las capacidades de los estudiantes, mediante la instrumentación de estrategias de organización de las competencias por estratos, es decir básicas y específicas, y orientadas hacia contenidos pragmáticos.

Esta situación permite reflexionar algunos hallazgos en el tema de las competencias, resulta complejo aceptar un concepto del término competencias, aun cuando esta supone la combinación de tres elementos: a) información, b) desarrollo de una habilidad y, c) puestos en acción en una situación inédita. Por lo tanto, la mejor manera de observar una competencia es en la combinación de estos tres aspectos, lo que significa que toda competencia, requiere del dominio de una información específica, al mismo tiempo que reclama el desarrollo de una habilidad o mejor dicho una serie de habilidades derivadas de los procesos de información, pero es en una situación problema, esto es, en una situación real inédita, donde la competencia se puede generar. (Díaz Barriga 2006).

En este aspecto, si bien la información aportada por el docente, a través de la plataforma está en clara coherencia con lo que pretende propiciar en la formación del estudiante, y al conjugar esto, con las asignaciones respectivas, habría que estimar que se incentiva a la puesta en marcha de una o varias habilidades, lo cierto es, que la situación presentada por el aislamiento social, limitada esta forma de educación mediante el uso de las Tic, debido a que no brinda oportunidades en los espacios virtuales, para insertar al estudiante en esta experiencia.

Con una situación bastante similar a lo descrito anteriormente, se presenta el indicador Aprendizaje analítico, de este se destaca, que es el de menor consideración para el docente como parte de su accionar pedagógico, en este proceso formativo, el estudiante, no está en la posibilidad de precisar la progresión de su desempeño, solo le es posible, conocer las calificaciones pero sin que ello promueva, una alternativa en la cual se involucre para la mejora de su situación académica, claro está, la dinámica acelerada de estos procesos, se concentra en actividades con periodos de consecución relativamente cortos, pero con un número significativo de estas por la cantidad de áreas de formación que debe atender.

Esta situación, determina una diferencia con el postulado que define al indicador, donde es evidente que se trata de un aprendizaje asistido por computador, o también llamado aprendizaje adaptativo, en este, se suministra un material al estudiante, para que a través de la plataforma pueda ejercitar y desarrollar experiencias, que contribuyan a su proceso de aprendizaje, así como, a la mejora de su rendimiento. (Gabinete de Comunicación y Educación de la Universidad Autónoma de Barcelona 2014).

También se identifica en la realidad del indicador Aprendizaje enfocado a la solución de problemas, una escasa posibilidad de incorporación del método dentro de la pedagogía tecnológica que ha de emprender el docente en la condición de virtualidad. Si bien las asignaciones que el docente establece dentro del proceso formativo, establecen 
no solo la lectura, estudio, comprensión, internalización y todo el resto de aspectos necesarios para el aprendizaje por parte de los estudiantes, también es cierto que, en muchos casos, el docente, identifica que los estudiantes, lejos de una dedicación con la actividad, han compartido la información con sus compañeros, al punto de replicar su ejecución y generar una copia, lo cual revela una situación, que agrede significativamente este complejo proceso formativo.

Los hechos descritos en la realidad pedagógica del docente difieren con el postulado teórico, en el cual se expone que, este método organiza las actividades con vistas a solventar un problema o una barrera para la consecución de unos objetivos previos, se trata en si de un sistema de análisis y de estrategia de acción, que todos deben asumir como un modo de pensamiento. (Gabinete de Comunicación y Educación de la Universidad Autónoma de Barcelona 2014).

De acuerdo a lo expresado sobre el indicador Aprendizaje por exploración, la realidad de los docentes, para con este método determina una escasa integración en la pedagogía tecnológica que ha de asumir en este proceso de formación apoyado en el uso de las Tic. Ciertamente, este es un método que tiene una clara especificación para llevarlo a la realidad del proceso educativo, por ello el docente, debe facilitar condiciones a los estudiantes para explorar la realidad, más aún con el uso de las tecnologías, y aun cuando en casos los docentes, logren recomendar la búsqueda de contenidos adicionales a los propuestos, la dinámica acelerada de los hechos determina que los estudiantes, no se encuentran en esa condición.

Tales situaciones, resultan contrarias al planteamiento de Bruner (1968), mentor del aprendizaje por exploración o descubrimiento, la cual constituye una teoría del aprendizaje de índole constructivista, por lo cual considera que los estudiantes, deben aprender mediante un descubrimiento guiado, el cual tiene lugar durante una exploración motivada por la curiosidad. De ahí que el objetivo final de este método radica en que los estudiantes logren descubrir cómo funcionan las cosas de un modo activo y constructivo, por tal motivo, el material proporcionado por el profesor constituye lo que Bruner denomina andamiaje.

De acuerdo con las experiencias de los docentes y su percepción, las realidades señaladas para el indicador Pedagogía inversa o Flipped Classroom, demuestran que es el único método en el cual se sitúan, debido a que son las instituciones donde laboran quienes han instrumentado este recurso, por ello, refieren que son gestores del trabajo con este método. Sin embargo, es válido considerar que, con toda la potencialidad que posee, si bien no ha sido aprovechada en buena medida por lo reciente del proceso, también es importante destacar que falta mayor voluntad de los actores para integrarse y alcanzar una educación de calidad.

Exponen por lo tanto estos resultados, una relación con las referencias teóricas al respecto, de ahí que este método, consiste en registrar las lecciones magistrales del profesorado en vídeo y distribuirlas a los estudiantes con anterioridad a las clases. De este modo, la actividad en el aula, no se concentra en la exposición y presentación magistral, sino en la discusión, reflexión o ejercitación de los contenidos, que se suponen ya adquiridos, para aquellos que se identifiquen que presentan dificultades, y logren trabajar 
de forma individual o grupal para resolverlos. (Gabinete de Comunicación y Educación de la Universidad Autónoma de Barcelona 2014).

\section{CONCLUSIONES}

Al concretar esta investigación, la cual para sus responsables ha permitido la adquision de experiencias y el poder concretar una serie de realidades, las cuales en muchas ocasiones quedan atrapadas, en la cotidianidad de los hechos que enmascaran la relevancia de la misma y por lo tanto, permiten un espectro cíclico de complejidades, se ha logrado conocer y analizar los puntos neurálgicos de la situación, los cuales deben servir como referencia explícita y base en la generación de cambios en el proceso educativo y una mejor instrumentación de la pedagogía tecnológica requerida en estos tiempos.

Considerando el aspecto de la Estrategia Metodológica, se hace evidente que en la oportunidad del ejercicio de la pedagogía que en todo caso debe ser tecnológica, por el cambio de paradigma, las diferentes alternativas existentes, no están debidamente instrumentadas en las instituciones de educación media, en las cuales se encuentran dispuestas plataformas de trabajo como el Classroom, aun cuando ha de comprenderse que son relativamente recientes los cambios de esta forma presencial a una virtualizada, las oportunidades de transformación del modelo, no han sido del todo impulsadas por las instituciones y en todo caso el docente, por sus limitaciones de distinta índole, no le resulta fácil poder asumirlo de manera particular.

En muchos casos, la falta de una correcta orientación hacia el docente ha subestimado su capacidad para brindarle espacio y mantenerlo en el mismo paradigma, pero con la utilización de la tecnología, en una manera instrumental y no productiva. De la misma forma, muchos docentes, consideran que algunas instituciones, han focalizado el trabajo pedagógico del docente, en interacciones sumamente básicas donde sus posibilidades para acudir al uso de las estrategias son escasas y en ocasiones resultan improvisadas no consolidando un resultado favorecedor.

Para el caso de los nuevos métodos pedagógicos, es sumamente claro que las posibilidades de asumirlos, está comprometida con el aspecto de la planificación que desde la perspectiva tecnológica, debe desarrollarse de manera conjunta entre el docente y la institución, pero hasta ahora, se ha continuado disponiendo de los mismos esquemas que se utilizaron para la educación presencial, por lo que al momento de hacer seguimiento, no existe una nueva orientación para con esta, y repetitivamente se improvisa la definición de las tareas tan solo con un cambio hacia el escenario de la tecnología, el cual por sí solo no garantiza un estilo tecnológico.

Otro de los aspectos que distancia la integración de estos métodos en la realidad pedagógica del docente, lo representa el interés que algunas instituciones tienen para 
aminorar una supuesta "complejidad de las actividades" que a juicio de los representes, saturan de trabajo al estudiante, ante esto realizan reclamos y hasta confrontaciones en la búsqueda que estas actividades escolares, sean sencillas, cortas y hasta individuales para evitar la oportunidad de tener que promover reuniones presenciales entre los compañeros.

Por encima de estas situaciones, es menester destacar el significativo papel del docente para superar las complejidades, haciendo esfuerzos que ciertamente no se encuentran debidamente compensados en el reconocimiento de su trabajo, la gran mayoría de ellos, sintieron el temor de no poder con el compromiso, pero son más los que han ganado la batalla para mantenerse constantes y dispuestos a aprender. Así mismo, existen instituciones, que han comprendido que la inversión necesaria debe iniciarla el Estado para democratizar un servicio de Internet, el cual se está haciendo posible para solo algunos que pueden cancelar en divisa extranjera, también estas instituciones, deben dar el justo reconocimiento al docente y fortalecer sus conocimientos, su condición socioeconómica, y al revitalizarlo será posible promover verdaderas transformaciones.

\section{REFERENCIAS BIBLIOGRÁFICAS}

Bittinger, M. (1968). A Review of Discovery. New York. The Mathematic Teacher

Bracho, K.J. \& Bracho, M. Ch., (2020). COVID-19: Facing the pedagogical challenge of physical attendance to virtual. Hamut'ay, 7 (2), 9-17. http://dx.doi.org/10.21503/ hamu.v7i2.2127

Bracho, Kleeder J. \& Bracho, Marianggeline Ch., (2020). COVID-19: Facing the pedagogical challenge of physical attendance to virtual. Hamut'ay, 7 (2), 9-17. http:// dx.doi.org/10.21503/hamu.v7i2.2127

Carranza-Marchena, P., y Zamora-Sánchez, G. (2020). Desafíos y oportunidades entiempos del COVID-19: contexto pedagógico desde la Universidad Nacional y la Universidad Estatal a Distancia. Innovaciones Educativas, 22(Especial), 162-170. DOI: https://doi.org/10.22458/ie.v22iespecial.3154 URL: https://revistas.uned. ac.cr/index.php/innovaciones/article/view/3154

Carrió, M.L. (2007). Ventajas del uso de la tecnología en el aprendizaje colaborativo. Revista Iberoamericana de Educación, 41, 1-10.

Díaz Barriga, Ángel. (2006). El enfoque de competencias en la educación: ¿Una alternativa o un disfraz de cambio? Perfiles educativos, 28(111), 7-36. Recuperado en 01 de abril de 2021, de http://www.scielo.org.mx/scielo.php?script=sci_arttext\&pid= S0185-26982006000100002\&lng=es\&tlng=es.

Espegel Alonso, C., Feliz Ricoy, S., y Buedo García, J. A. (2020), Poliesferas Pedagógicas. Estudio analítico de las cosmologías locales del Covid-19. DOI: 10.5821/jida.2020.9356.

Galindo Cuervo, Nicolas y Vela Palacios, Jaime (2020) Motivación académica en tiempos de covid-19, de estudiantes vinculados a universidades de villavicencio: a partir de 
la teoría de Deci y Ryan. Universidad Santo Tomás Facultad de psicología Villavicencio. Colombia.

García-Valcárcel, A. y Tejedor, J. (2010). Evaluación de procesos de innovación escolar basados en el uso de las TIC desarrollados en la Comunidad de Castilla y León. Revista de Educación, 352, 125-148.

Hernández Sampieri, R., Fernández Collado, C., \& Baptista Lucio, P. (2014). Metodología de la investigación (6a. ed. --.). México D.F.: McGraw-Hill.

Lee, S.W., \& Tsai C.C. (2013). Tecnologysupported learning in Secondary and Undergraduate Biological Education: Observations form literature. Rewiew. Journal of Science Education and Tecnology, 22, 226-233

Manrique, Diamire (2020, abril). De la Pedagogía a la Tecno-pedagogía en días, por la pandemia del COVID-19, el teletaller como estrategia metodológica para la sostenibilidad de la formación virtual por proyectos formativos desde la socioformación. En J. Luna- Nemecio (Coord.), Memorias del Quinto Congreso Internacional de Investigación en Socioformación y Sociedad del Conocimiento (CISFOR-2020). Congreso conducido por el Centro Universitario CIFE, Cuernavaca, México. Descarga de: https://cife.edu.mx/recursos

Michel Salazar, José A. (2006). Sobre el estatuto epistemológico de las ciencias de la educación. Revista de Teoría y Didáctica de las Ciencias Sociales, (11),139-157. [fecha de Consulta 30 de Marzo de 2021]. ISSN: 1316-9505. Disponible en: https:/ / www.redalyc.org/articulo.oa?id $=65201107$

Peñafiel Rodríguez, W. (2020). Artículo Editorial Los cambios en los procesos educativos en tiempos de pandemia. Fides et Ratio-Revista de Difusión cultural y científica de la Universidad La Salle en Bolivia, 20(20), 13-16.

Plomp, T. y Voogt, J. (2009). Pedagogical practices and ICT Use around the world: Findings from the IEA International Comparative Study SITES 2006. Education and Information Technologies, 14(4), 285-292.

Prieto, F., Rodríguez, L., \& Verdugo, R. (2020). Fortalezas y debilidades de la pedagogía en el ciberespacio en época de cuarentena COVID-19. Revista Scientific, 5(17), 252266, e-ISSN: 2542-2987. Recuperado de: https://doi.org/10.29394/Scientific.issn. 2542-2987.2020.5.17.13.252-266

Vialart Vidal, M. N. (2020). Estrategias didácticas para la virtualización del proceso enseñanza aprendizaje en tiempos de COVID-19. Educación Médica Superior, 34(3).

Vidal Ledo M, Gavilondo Mariño X. (2018) Docencia y tecnologías móviles. Educ Med Super. 2018 [acceso 17/07/2020];32(2). Disponible en: http://scielo.sld.cu/scielo. php?script=sci_arttext\&pid=S0864-21412018000200027\&lng $=$ es 